\title{
A Bimetallic, Heterogeneous, Multifunctional Catalyst
}

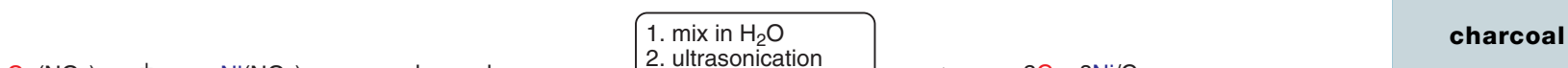

isolable, non-pyrophoric $>20 \mathrm{~g}$ scale

2Cu-8Ni/C $(2.5 \mathrm{~mol} \% \mathrm{Ni})$ $\mathrm{PPh}_{3}(15 \mathrm{~mol} \%)$

$\mathrm{Ar}^{1} \mathrm{Cl}(\mathrm{Br})$

$(\mathrm{HO})_{2} \mathrm{BAr}^{2}$

$\mathrm{KF}$ (3 equiv), LiOH (5 equiv)

$\operatorname{Ar}^{1} \mathrm{Ar}^{2}$

78-92\% (5 examples)

1

2

dioxane, MW

3

$180-200{ }^{\circ} \mathrm{C}, 66-75 \mathrm{~min}$

2Cu-8Ni/C (10 mol\% Ni)

$\begin{array}{cc}\mathrm{Ar}^{3} \mathrm{Cl} & + \\ 4 & \mathrm{RR}^{\prime} \mathrm{NH} \\ & 5\end{array}$

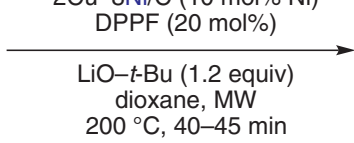

$\mathrm{Ar}^{3} \mathrm{NRR}^{\prime}$

79-96\% (8 examples)

$200^{\circ} \mathrm{C}, 40-45 \mathrm{~min}$

6

$2 \mathrm{Cu}-8 \mathrm{Ni} / \mathrm{C}(10 \mathrm{~mol} \% \mathrm{Ni})$
$\mathrm{PPh}_{3}(20 \mathrm{~mol} \%)$

$\mathrm{Ar}^{4} \mathrm{Cl}+\mathrm{BH}_{3} \cdot \mathrm{HNMe}_{2}$

8

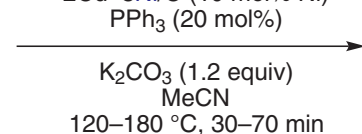

$\mathrm{Ar}^{4} \mathrm{H}$

92-100\% (3 examples)

7

$120-180{ }^{\circ} \mathrm{C}, 30-70 \mathrm{~min}$<smiles>ClC[18Cl]</smiles>

10

$\mathrm{R}^{\prime \prime} \mathrm{N}_{3}$

13

$\mathrm{Ar}^{6} \mathrm{Br}$

16<smiles>CC(=CC(C)C)c1ccccc1</smiles>

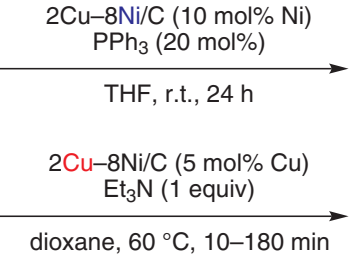

14

2Cu-8Ni/C (8 mol\% Cu) phenanthroline (50 mol\%)

$\mathrm{HO}-\mathrm{Ar}^{7}$

17

$\mathrm{Cs}_{2} \mathrm{CO}_{3}$ (1.2 equiv) dioxane, MW
$200^{\circ} \mathrm{C}, 30-60 \mathrm{~min}$

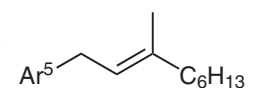

76-85\% (3 examples)

12<smiles>CCn1cc(Br)nn1</smiles>

89-99\% (4 examples) 15 R"' $\mathrm{Ar}^{6}-\mathrm{O}^{-} \mathrm{Ar}^{7}$

78-95\% (5 examples)

18

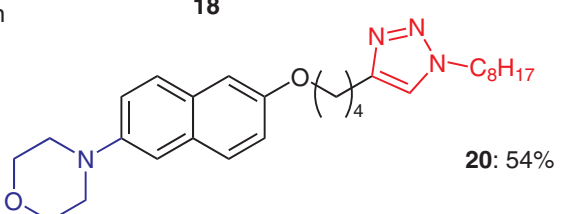

Significance: A bimetallic heterogeneous catalyst, 2Cu-8Ni/C, of copper and nickel oxide particles in charcoal was prepared. The resulting catalyst promoted a variety of cross-couplings typically associated with group 10 and 11 transition metal catalysts. The catalyst was reused once without loss of catalytic activity.
Comment: More than one metal are housed in the solid support, and hence, the resulting catalyst mediates multiple types of bond constructions. A tandem click reaction-amination of $\mathbf{1 9}$ was carried out with the multifunctional catalyst to give the coupling product 20 in 54\%.

SYNFACTs Contributors: Yasuhiro Uozumi, Yoichi M. A. Yamada DOI: 10.1055/s-0028-1083554; Reg-No.: Y12508SF 